\title{
Study of cranial ultrasound its correlation with perinatal risk factors and its outcome in preterm neonates admitted to Neonatal intensive care unit
}

\author{
Kinikar U. ${ }^{1}$, Dhanawade S. ${ }^{2}$ \\ ${ }^{1}$ Dr. Utkarsha Kinikar, Resident, ${ }^{2}$ Dr. Sara Dhanawade, Professor, both authors are affiliated with Department of \\ Pediatrics, Bharati Vidyapeeth Deemed University Medical College \& Hospital, Sangli, Maharashtra, India.
}

Address for Correspondence: Dr. Sara Dhanawade, Professor and HOD, Department of Pediatrics Bharati Vidyapeeth Deemed University Medical College \& Hospital, Sangli.

E-mail: kurade.aditya@gmail.com, utkarshakinikar@rediffmail.com

\begin{abstract}
Aims and Objectives:To determine the frequency of cranial ultrasound abnormalities in preterm neonates in the first week of life. To evaluate the association between cranial ultrasound findings and perinatal risk factors in regard to the immediate clinical outcome. Materials and Methods: This Retrospective study was done in NICU in a tertiary hospital. Records of all preterm neonates ( $<37$ weeks) admitted to NICU who underwent neuro-sonography between Jan2016 - Jan 2017 were included. Results: Out of 100 preterms, 53\% had normal CUS findings while 47\% had abnormal finding. Abnormal CUS was significantly associated with gestational age $<32$ weeks $(\mathrm{p}<0.001)$ and birth weight $<1.5 \mathrm{~kg}$ $(\mathrm{p}=0.006)$. Among the abnormal CUS findings intraventricular hemorrhage was the most common $(40.42 \%)$ followed by periventricular hyper-echogenicity $(21.27 \%)$, cystic periventricular leukomalacia $(8.51 \%)$, parenchymal bleed $(8.51 \%)$, cysts $(8.51 \%)$, cerebral edema $(6.38 \%)$, ventriculomegaly $(4.25 \%)$ and thalamic injury $(2.12 \%)$. Neonatal comorbidities associated with abnormal cranial ultrasound were RDS $(25.53 \%)$, neonatal sepsis $(21.27 \%)$, birth asphyxia (17.02\%), neonatal seizures $(8.51 \%)$, NEC $(6.38 \%)$ and others $(21.27 \%)$. There was significant association between abnormal cranial ultrasound and RDS ( $\mathrm{p}=0.014)$ and birth asphyxia $(\mathrm{p}=0.008)$. Mortality was $36.1 \%$ and all had abnormal cranial USG. Intraventricular hemorrhage $(76.4 \%)$ was the most common cause of death followed by parenchymal bleed (11.7\%). Conclusion: There was significant association between abnormal CUS and gestational age and birth weight. Most common abnormality was IVH and it carried the highest mortality. RDS and birth asphyxia had significant association with abnormal ultrasound. Early neurosonography could help in prognosticating immediate outcome and early intervention.
\end{abstract}

Keywords: Preterms, Cranial USG, Intraventricular hemorrhage

\section{Introduction}

Preterm neonates have increased risk of birth asphyxia, respiratory distress syndrome, temperature instability, hypoglycemia and sepsis [1]. Premature neonate is also vulnerable to both hemorrhagic and ischemic brain injuries. This is due to vascular, cellular and anatomical feature of developing brain and physiological instability because of limited cerebral auto regulation $[1,2]$.

Germinal matrix hemorrhage (GMH) / Intraventricular hemorrhage (IVH) and white matter injury particularly cystic periventricular leukomalacia (cPVL) are major

Manuscript received: $14^{\text {th }}$ March 2018

Reviewed: $24^{\text {th }}$ March 2018

Author Corrected: $30^{\text {th }}$ March 2018

Accepted for Publication: $4^{\text {th }}$ April 2018 contributors to mortality and morbidity [3]. Although the mortality rates have fallen down due to improvement in obstetrics and neonatal intensive care, there is growing concern over adverse neuro developmental outcome. Almost $10-15 \%$ preterm neonates develop cerebral palsy while upto $50 \%$ have another adverse neurological outcome[4].

Cranial ultrasound (CUS) has become an essential diagnostic tool in neonatology as it can depict normal anatomy and pathological changes in neonatal brain. Cranial ultrasound is a technique for scanning the brain using high-frequency sound waves through their fontanelle, which provides an "acoustic window"[5,6,7]. 
Cranial ultrasound detects most of hemorrhagic, ischemic and cystic brain lesion as well as calcification, cerebral infarction and major structural anomalies congenital or acquired [5]. It is very useful in early diagnosis, assessing severity and neurodevelopmental outcome in hypoxic-ischemic encephalopathy (HIE). In seriously ill neonates with cerebral abnormality it plays a role in decision on continuation or with drawal of intensive treatment. In preterm neonates surviving with cerebral injury, it may help to optimize treatment in neonatal period and even thereafter[5,6,8,9].

It is cheap, easy to perform, non-invasive and radiation free. It can be initiated at very early stage, even immediately after birth and is repeatable without any adverse effect. Serial cranial ultrasound enables assessment of onset of injury and evolution of lesion.

Early identification of brain abnormalities by using cranial ultrasound would allow early interventions to improve long-term outcome. However cranial ultrasound has limitations as quality of imaging depends upon skills and experience of ultrasonographer, some areas are difficult to visualize and several abnormalities remain beyond its scope[6,8].

Routine screening CUS in general are recommended for all infants born before 34 weeks gestational, but higher brain vulnerability in late preterm infants as compared to term babies is well known [1]. Early identification of late preterms with brain abnormalities at CUS would allow early intervention programme to improve longterm outcomes. In our newborn care unit routine cranial USG are performed in all preterm infants early in the course of hospitalization and then follow up at later age.
This retrospective study was aimed to evaluate the abnormalities detected in early neurosonography and their correlation with perinatal risk factors and clinical outcome.

\section{Materials and Methods}

Study design: Retrospective analysis study. Study area: Neonatal intensive care unit (NICU) of a tertiary care teaching hospital in western Maharashtra.

Study period: January 2016 to January 2017.

Inclusion criteria: All Preterm infants ( $<37$ weeks gestational age) who had cranial ultrasound performed in the first week of life were included in the study.

Exclusion criteria: Incomplete data.

Study methodology: Database of all admissions in NICU during the above period were reviewed. Case records of all preterm babies admitted were analyzed. According to the protocol of our unit cranial USG were routinely performed on day 3 , day 7 , at 2 weeks and at 40 weeks of corrected age. In the present study we have looked at the early cranial USG abnormalities. All CUS were performed by single radiologist. The cranial ultrasound findings, other demographic parameters, perinatal risk factors, clinical status and immediate outcome were obtained from the case records.

Statistical analysis- Continuous variables were summarized using means, while categorical variables were summarized using percentages. The Chi-square test was used to determine the association of different variables.

\section{Results}

Out of 100 preterm neonates, 93 had CUS on day 3 and day 7 while 7 neonates had only cranial ultrasound in the $1^{\text {st }}$ week of life.

Thirty-one (65.96\%) neonates with gestational age $<32$ weeks and $16(34.04 \%)$ neonates with gestational age $>32$ weeks had abnormal neurosonogram. Thirty $(63.8 \%)$ neonates with birth weight $<1.5 \mathrm{~kg}$ and 17 neonates $(36.17 \%)$ with birth weight $>1.5 \mathrm{~kg}$ had abnormal CUS. Abnormal CUS was significantly associated with gestational age $<32$ weeks $(\mathrm{p}<0.001)$ and birth weight $<1.5 \mathrm{~kg}(\mathrm{p}=0.006)$ (Table 1$)$.

Table-1: Distribution according to gestational age and birth weight

\begin{tabular}{|c|c|c|c|c|}
\hline & & Normal & Abnormal & \multirow{2}{*}{ P value } \\
\hline \multirow{2}{*}{$\begin{array}{c}\text { Gestational age } \\
\text { (Weeks) }\end{array}$} & $<=32$ & $10(24.39 \%)$ & $31(75.60 \%)$ & \multirow{2}{*}{$<0.001$} \\
\cline { 2 - 4 } & $>32$ & $43(76.78 \%)$ & $16(34.04 \%)$ & \\
\hline \multirow{2}{*}{ Birth weight (Kg) } & $<=1.5$ & $22(41.5 \%)$ & $30(63.8 \%)$ & \multirow{2}{*}{$\mathbf{0 . 0 0 6}$} \\
\cline { 2 - 4 } & $>1.5$ & $31(58.4 \%)$ & $17(36.17 \%)$ & \\
\hline
\end{tabular}


Among the abnormal CUS findings intraventricular hemorrhage was the most common (40.42\%) followed by periventricular hyper-echogenicity $(21.27 \%)$, cystic periventricular leukomalacia $(8.51 \%)$, parenchymal bleed $(8.51 \%)$, cysts $(8.51 \%)$, cerebral edema $(6.38 \%)$, ventriculomegaly $(4.25 \%)$ and thalamic injury $(2.12 \%)$. (Table 2$)$ The four cases with cystic periventricular leukomalacia were confirmed on magnetic resonance imaging.

Table-2: Distribution according to abnormal CUS findings

\begin{tabular}{|c|c|c|}
\hline Abnormal Findings & $\begin{array}{c}\text { Frequency } \\
\mathbf{n = 4 7}\end{array}$ & $\begin{array}{c}\text { Percentage } \\
\mathbf{( 1 0 0 \% )}\end{array}$ \\
\hline IVH & 19 & 40.42 \\
\hline PHE & 10 & 21.27 \\
\hline cPVL & 4 & 8.51 \\
\hline Parenchymal bleed & 4 & 8.51 \\
\hline Cysts & 4 & 8.51 \\
\hline Cerebral edema & 3 & 6.38 \\
\hline Ventriculomegaly & 2 & 4.25 \\
\hline Thalamic injury & 1 & 2.12 \\
\hline
\end{tabular}

IVH-Intraventricular hemorrhage, PHE- Periventricular hyper-echogenicity, cPVL- Cystic periventricular hemorrhage

Maternal risk factors present in neonates with abnormal CUS were PIH (53.1\%), PROM (25.5\%), APH (8.5\%) and others (12.7\%) (Table 3) However there was no significant association.

Table-3: Distribution according to maternal risk factors

\begin{tabular}{|c|c|c|c|}
\hline Maternal Risk Factors & $\begin{array}{c}\text { Normal Cranial USG } \\
\mathbf{n = 5 3}\end{array}$ & $\begin{array}{c}\text { Abnormal Cranial USG } \\
\mathbf{n = 4 7}\end{array}$ & P value \\
\hline PIH & $26(49 \%)$ & $25(53.1 \%)$ & 0.679 \\
\hline PROM & $17(32 \%)$ & $12(25.5 \%)$ & 0.471 \\
\hline APH & $4(7.5 \%)$ & $4(8.5 \%)$ & 0.859 \\
\hline Other & $6(11.3 \%)$ & $6(12.7 \%)$ & 0.824 \\
\hline
\end{tabular}

PIH- Pregnancy induced hypertension, PROM- Premature rupture of membrane, APH- Antepartum hemorrhage

Neonatal comorbidities associated with abnormal USG were RDS (25.53\%), neonatal sepsis (21.27\%), birth asphyxia $(17.02 \%)$, neonatal seizures $(8.51 \%)$, NEC (6.38\%) and others $(21.27 \%)$. (Table 4) There was significant association between abnormal CUS and RDS $(\mathrm{p}=0.014)$, birth asphyxia $(\mathrm{p}=0.008)$.

Table-4:Distribution according to neonatal comorbidities

\begin{tabular}{|c|c|c|c|}
\hline Neonatal Comorbidities & $\begin{array}{c}\text { Normal Cranial USG } \\
\mathbf{n = 3 5}\end{array}$ & $\begin{array}{c}\text { Abnormal cranial USG } \\
\mathbf{n = 4 7}\end{array}$ & P value \\
\hline RDS & $4(10.52 \%)$ & $12(25.53 \%)$ & $\mathbf{0 . 0 1 4}$ \\
\hline Neonatal Sepsis & $5(13.15 \%)$ & $10(21.27 \%)$ & 0.097 \\
\hline Birth Asphyxia & $1(2.63 \%)$ & $8(17.02 \%)$ & $\mathbf{0 . 0 0 8}$ \\
\hline Neonatal Seizures & $1(2.63 \%)$ & $4(8.51 \%)$ & 0.129 \\
\hline NEC & $2(5.26 \%)$ & $3(6.38 \%)$ & 0.55 \\
\hline Others & $25(47.16 \%)$ & $10(21.27 \%)$ & \\
\hline
\end{tabular}

RDS- Respiratory distress syndrome, NEC- Necrotizing Enetrocolitis

Mortality was $36.1 \%$ and all had abnormal cranial USG findings. Intraventricular hemorrhage (76.4\%) was the most common cause of death followed by parenchymal bleed in $11.7 \%$. 


\section{Discussion}

Cranial ultrasonography is currently the primary imaging modality employed in the NICU for assessment of neonatal brain. While Daneman et al reported cranial ultrasound to be extremely useful modality in neonates, De Vries and Cowan et al have suggested that cranial ultrasound and MRI brain are complementary modalities [10,11]. Benefits of CUS are that it can be performed at the bedside and in the sickest infant. It allows early detection of congenital as well as hypoxicischemic brain injury. When neonate is unstable for transport, cranial USG findings may be sufficient for major clinical decisions $[11,12,13]$.

The incidence of early cranial ultrasound abnormalities was $47 \%$ in the present study. Our results are comparable to Brad way et al study who reported $37 \%$ abnormality in early sonography [14]. Most of the other Indian reports have reported incidence of $25.45 \%$, $29.8 \%, 36 \%, 38 \%[5,6,8,15]$. However these studies have not specified the exact time of neurosonography. Higher incidence of abnormal cranial ultrasounds in our study could probably be explained on fact that we have included findings of neurosonography in first week of life. It is possible that some abnormalities would have resolved on follow up.

In the study there is significant association between abnormal cranial USG and gestational age and birth weight. Similar observations were also reported by Fumagalli et al and Jha et al in their study[1,15].

Among infants who develop GMH and/or IVH, at least one third of them develop echo-density as early as onehour indicating antenatal or immediate post-natal onset. Approximately $50 \%$ of IVH occur within first 6-8 hours, most of them evident by third day but can develop anytime in first two weeks [2]. It has been suggested that CUS performed in second week provides the most complete and reliable diagnosis regarding hemorrhagic lesions $[2,16]$.

Although white matter abnormalities, ventriculomegaly and cysts may be present at birth they often appear later. Cystic PVL of antenatal origin is evident by two weeks of age[2]. The evolution of postnatally acquired periventricular leukomalacia cysts are evident within 60 days of birth. Repeated ultrasounds should be performed as clinically indicated, in infants with identified brain injuries. Early cranial ultrasound may be of more prognostic value in early onset IVHs. However, if only one ultrasound examination is to be performed to predict long-term neurodevelopmental outcomes, the most appropriate time is two weeks after birth. Early ultrasound examination allows diagnosis of hemorrhagic lesions and later CUS can detect white matter abnormalities, ventriculomegaly and cysts[2].

In the present study intraventricular hemorrhage (40.42\%) was the most common finding followed by periventricular hyper-echogenicity (21.27\%).

Previous studies by Bradway et al and Prithviraj et al also reported similar findings with incidence of intraventricular hemorrhage to be $38 \%$ and $43 \%$ respectively [8,14]. Nagraj et al and Jha et al, two Indian studies reported periventricular echogenicity as the most common finding[5,15].

PIH was the most common maternal risk factor associated with abnormal cranial USG followed by PROM. But the association between maternal risk factors and abnormal neurosonogram were not statistically significant in the present study.

Similar observation was reported by Nagraj et al in their prospective study of perinatal risk factors and cranial ultrasound findings [17]. Prithviraj et al reported that correlation of abnormal cranial USG with PIH was significant, but other maternal risk factors were statistically not significant [8]. Vermeulen GM et al observed that $13 \%$ neonates with abnormal cranial ultrasound had PROM as the maternal risk factor[18].

In our study there was significant association between neonatal comorbidities like RDS, Birth asphyxia and abnormal neurosonogram. Most common abnormality was RDS (22\%) followed by neonatal sepsis (20.33\%) and birth asphyxia (13.5\%). Fumagalli et al also observed increased risk of brain injury mainly IVH in preterm neonates with RDS as it has been associated with fluctuation of cerebral blood flow in the first few days of life [1]. Vermeulen et al reported early onset neonatal infectious disease as an independent risk factor for abnormal cranial USG[18].

Mortality was $36.1 \%$ in abnormal cranial USG group while none died in the group with normal CUS. Intraventricular hemorrhage was the most common cause of mortality in the study. This is consistent with findings of Ghooret al [3]. Ballabh $\mathrm{P}$ et al and Mulindwa MJ et al also reported that birth asphyxia, sepsis, mechanical ventilation, PDA were associated 
with increased risk of IVH. They also reported higher mortality in infants with intraventricular hemorrhage $[19,20]$. CUS can provide as a safe and effective diagnostic tool for IVH and ischemic periventricular damage in preterm infants. However when testing performed early in life for the purpose of predicting long term outcome one should keep in mind false positive and false negative results when communicating the test results to the parents.

The major use of early testing is to direct the families of affected infants towards proper follow up for early diagnosis and promote early intervention. The major limitation of this study was the retrospective nature.

\section{Conclusion}

There was significant association between abnormal cranial ultrasound and gestational age $<32$ weeks and birth weight $<1.5 \mathrm{~kg}$. Most common abnormality was intraventricular hemorrhage and it carried the highest mortality. RDS and birth asphyxia had significant association with abnormal CUS. Early neurosonography could help in prognosticating immediate outcome and early intervention.

What is already known- Cranial ultrasound is an effective diagnostic tool for Intraventricular hemorrhage and ischemic periventricular damage in preterm infants.

What this study adds? - Early neurosonography is useful in prognosticating immediate outcome.

Acknowledgement: Alka Gore for assistance in statistical analysis.

Contribution: UK: Data collection, statistical analysis, literature search and manuscript preparation. SD: Study concept and critical revision of manuscript.

Funding: Nil, Conflict of interest: None initiated, Perission from IRB: Yes

\section{Reference}

1. Fumagalli M, Ramenghi LA, De Carli A, et al. Cranial ultrasound findings in late preterm infants and correlation with perinatal risk factors. Ital $\mathrm{J}$ Pediatr2015;41:65. doi:10.1186/s13052-015-0172-0.

2. Canadian Paediatric Society Statement. Routine screening cranial ultrasound examinations for the prediction of long-term neurodevelopmental outcomes in preterm infants. Paediatr Child Health. 2001; 6(1): $39-43$.
3. Ghoor A, Scher G, Ballot DE. Prevalence of and risk for cranial ultrasound abnormalities in very-low-birthweight infants at Charlotte Maxeke Johannesburg academic hospital.SAfr JChild Health 2017;11(2):66-70

4. Thompson DK, Warfield SK, Carlin JB, et al. Perinatal risk factors altering regional brain structure in preterm infant. Brain 2007;130(3):667-677. Doi:10. 1093 /brain/awl277.

5. Nagraj N, Berwal PK, Srinivas A, et al. A study of neurosonogram abnormalities, clinical correlation with neurosonogram findings, and immediate outcome of high-risk neonate in neonatal intensive care unit. J PediatrNeurosci2016;11:200-5.

6. Poornima Shankar, Nithya S.L. Role of cranial ultrasound in high risk neonates in NICU. $J$ of Evolution Med and Dent Sci 2014;3(15);3970-3976.

7. Gerda van Wezel-Meijler. Cranial Ultrasonography: advantages and aims part 1, Neonatal Cranial ultrasonography, $1^{\text {st }}$ edition. Berlin: Springer, 2007: pg 3-4

8. Prithviraj D, Reddy B, Shruthi B. Neurosonogram in critically ill neonates in neonatal intensive care unit. Int J Sci Stud 2016;4(1):124-128.

9. de Vries LS, Groenendaal F. Neuroimaging in preterm infants. Ment Retard Dev DisabilRes Rev 2002; 8(4): 273-80. Doi:10.1002/mrdd.10050.

10. Daneman A, Epelman M, Blaser S, Jarrin JR. Imaging of the brain in full-term neonates: does sonography still play a role? PediatrRadiol. 2006 Jul; 36 (7):636-46. Epub 2006 May 16.

11. de Vries LS, Cowan FM. Should cranial MRI screening of preterm infants become routine? Nat Clin Pract Neurol. 2007 Oct;3(10):532-3. Epub 2007Aug 28.

12. Tann CJ, Nakakeeto M, Hagmann C, et al. Early cranial ultrasound findings among infants with neonatal encephalopathy in Uganda: an observational study. Pediatric RESEARCH 2016;80(2):190-196.

13. Leijser LM, Vein AA, Liauw L, Strauss T, Veen S, Wezel-MeijlerGv. Prediction of short-term neurological outcome in full-termneonates with hypoxic-ischaemic encephalo-pathybased on combined use of electro encephalogram and neuro-imaging. Neuropediatrics. 2007 Oct;38(5):219-27. doi: 10.1055 /s- 2007-992815. 
14. Bradway N, Edrees A, Sebaie DE, et al. Cranial ultrasonographic screening of the preterm newborn. Alex J Pediatr 2005;19(2):347-356.

15. Jha R, Singh A, Jha R. Cranial ultrasound in high risk preterm. New Indian Journal of Pediatrics 2017; 6:26-32.

16. Boal DK, Watterberg KL, Miles S, et al. Optimal cost-effective timing of cranial ultrasound screening in low birth weight infants. Pediatr Radiol 1995;25 (6): 425-8. PubMed PMID: 7491190.

17. Nagraj N, Berwal PK, Choudhary S. A prospective study of correlation of gestational age, birth weight and perinatal risk factors with various cranial ultrasound findings in high risk neonates. Indian Journal of Neurosciences 2016;2(2):43-45.
18. Vermeulen GM, Bruinse HW, Gerards LJ, et al. Perinatal risk factors for cranial ultrasound abnormalities in neonates born after spontaneous labour before 34 weeks. Eur J Obstetrics Gynecology Reprod Biol 2001; 94(2):290-295.

19. Ballabh P. Pathogenesis and prevention of intraventricularhemorrhage. Clin Perinatol. 2014 Mar; 41 (1): 47-67. doi: 10.1016/j.clp.2013.09.007. Epub 2013 Dec 12.

20. Mulindwa MJ, Sinyangwe S, Chomba E. The prevalence of intraventricular hemorrhage and associated risk factors in preterm neonates in the neonatal intensive care unit at the University teaching hospital, Lusaka, Zambia. Med J Zambia 2012; 39 (1): $1-6$.

\section{How to cite this article?}

Kinikar U, Dhanawade S. Study of cranial ultrasound its correlation with perinatal risk factors and its outcome in preterm neonates admitted to Neonatal intensive care unit. Int J Pediatr Res. 2018;5(4):169-174. doi:10.17511/ijpr.2018.i04.03. 